\title{
High resolution and uniform image reconstruction in a large field-of- view for EUV actinic mask review
}

\author{
H. Kim, U. Locans, R. Nebling, A. Dejkameh, D. Kazazis, Y. Ekinci, and I. Mochi \\ Paul Scherrer Institute (PSI), Switzerland \\ Contact author: hyun-su.kim@psi.ch
}

\begin{abstract}
Actinic EUV mask metrology is essentially needed for EUV lithography in the semiconductor device manufacturing process. At PSI, we are developing RESCAN, a coherent diffractive imaging (CDI)-based platform that can meet current and future mask inspection resolution requirements. In CDI, the diffraction patterns obtained by illuminating the sample with coherent light are recorded by a pixel detector, and these are used to reconstruct the complex-amplitude image of an object through an iterative phase retrieval algorithm. While in a conventional optical system, aberrations can compromise the final image's resolution, the CDI approach is inherently aberration-free. Nevertheless, a careful preprocessing of the diffraction signal is necessary to avoid artifacts in the reconstructed image. In particular, since our system works in reflection mode with an angle of incidence of $6^{\circ}$ and uses a flat detector, it is necessary to correct the recorded diffraction patterns that are conically distorted due to the non-telecentricity. This paper discusses the impact of the diffraction data preprocessing on the reconstructed image quality and demonstrates the defect sensitivity improvement by applying an optimized data preprocessing pipeline in the RESCAN microscope. As a result, we achieve defect sensitivity down to 20 $\mathrm{nm}$ on the photomask and uniform image quality in a large field-of-view.
\end{abstract}

Keywords: Extreme ultraviolet, defect inspection, lensless imaging, ptychography, actinic, photomask, mask review

\section{INTRODUCTION}

For high-yield manufacturing and better quality of semiconductor chips, actinic patterned mask inspection (APMI) is an essential process in the extreme ultraviolet lithography (EUVL) ecosystem that is needed from the production to the deliveries on chucks. The feature sizes that have to be inspected and reviewed are becoming smaller with advanced mask materials. For these reasons, continuous development of the inspection technology is necessary to enable further progress of EUVL for future technology nodes [1-4].

RESCAN (Reflective-mode Scanning microscope) is a synchrotron-based APMI technology platform operating at the XIL-II beamline of the Swiss light source at PSI [5, 6]. It is capable of actinic inspections for both phase and amplitude defects on EUV photomasks [7]. It allows for detecting defects down to a size of about $50 \times 50 \mathrm{~nm}^{2}$ [8]. Also, throughpellicle imaging was demonstrated in RESCAN last year [9]. We currently plan an upgrade of the tool towards $<20 \mathrm{~nm}$ defect resolution to meet the $5 \mathrm{~nm}$ EUVL technology node and below [10].

The working principle of RESCAN is based on coherent diffractive imaging (CDI), which is a lensless imaging method [11]. Accordingly, RESCAN uses a diffraction recording detector and reconstruction algorithm instead of lenses to obtain the aerial image of the EUV mask. We also implement and develop the algorithm by considering various ptychography approaches to optimize the reconstruction speed and accuracy [12]. Ptychography is a scanning CDI technique that allows for the reconstruction of large field objects. Even though the probe size confines each detection field at every scan position, with the proper compromise between probe overlap and probe size, a large field of view (FOV) can be obtained.

By the physical nature of the reflection mode and the difficulty of using beam splitters at EUV wavelength, an angle between the sample and detector planes is unavoidable, and the imaging is non-telecentric similar to the imaging conditions in the EUV scanners. The reconstructed image distortion caused by the tilt is often neglected. However, as the feature size decreases, it becomes more critical for the image quality and defect sensitivity.

Photomask Technology 2020, edited by Moshe E. Preil, Proc. of SPIE Vol. 11518, 115180X · @ 2020 SPIE · CCC code: 0277-786X/20/\$21 · doi: 10.1117/12.2573240 
In this paper, we show and discuss the impact of the distortion of the diffraction patterns on the reconstructed aerial images and the benefits of using a diffraction correction algorithm resulting in improved image quality and thereby smaller defect sensitivity. It also removes the focus gradient observed in reconstructed images and increases the FOV.

\section{MATERIALS AND METHODS}

\section{RESCAN specifications}

RESCAN is a synchrotron-based platform dedicated to actinic EUV mask review. Specifications of the current RESCAN tool are summarized in Table 1. The central wavelength is tunable $\pm 10 \%$ around $13.5 \mathrm{~nm}$, which is limited by the optics. The beam generated by an undulator is spatially filtered by the pinhole and has temporal coherence of $\lambda / \Delta \lambda=1500$ after the monochromator. The EUV beam is focused by a multilayer-coated spherical mirror onto the EUV mask. The incident angle on the mask is $6^{\circ}$ from the surface normal. The full collection NA is around 0.22 in the setup, but in this study, we use the CCD length of around $90 \%$, which leads to NA $=0.19$.

Table. 1. Specifications of the current version of the RESCAN tool

\begin{tabular}{|c|c|c|c|}
\hline Wavelength & $13.5 \mathrm{~nm}( \pm 0.1 \mathrm{~nm})$ & Sample size & $20 \times 20 \mathrm{~mm}^{2}$ \\
\hline$\lambda / \Delta \lambda$ & 1500 & Inspect field area & $200 \times 200 \mu \mathrm{m}^{2}$ \\
\hline Flux & $10^{12}($ photons $/ \mathrm{s})$ & Resolution (pitch) & $<76 \mathrm{~nm}$ \\
\hline Detector pixels & $2048 \times 2048$ & $\Delta x$ (Eqn.(1)) & $<38 \mathrm{~nm}$ \\
\hline Detector size & $27.6 \times 27.6 \mathrm{~mm}^{2}$ & Sensitivity for & $5 \mathrm{~nm}$ node \\
\hline
\end{tabular}

\section{Resolution and sensitivity of RESCAN}

In the setup, the scattered light from the sample to the detector has NA $=\sin (\arctan (L / 2 D))$, where $L=27.6 \mathrm{~mm}$ is the detector size and $D=62 \mathrm{~mm}$ is the distance from the sample to the center of the detector. Therefore, the theoretical detectable defect size, $\Delta x$, can be defined as

$$
\Delta x=k \cdot 0.6 \frac{\lambda}{N A}=\frac{k \cdot 0.6 \cdot \lambda}{\sin \left(\tan ^{-1}(L / 2 D)\right)}
$$

which results in $\Delta x=37.5 \mathrm{~nm}$ for the present configuration of the RESCAN tool.

The resolution can be improved (to some extent) by using a larger detector with the same pixel size or by reducing the distance D with the smaller pixel size to increase the NA. In a given optical system, the process-related factor, $k$ plays a substantial role in resolution to approach the theoretical limit. The most dominant aspect to obtain the minimum $k$ includes the algorithm in the sense of how to utilize the collected diffraction data.

The different defect shapes yield different signals on the reconstructed image even though the defect size remains the same. Accordingly, we test RESCAN with various defect shapes placed on different locations on the mask pattern. In the Results section, we will show an example of the defect signal with different defect sizes for a particular defect type. 
(1) ref mask

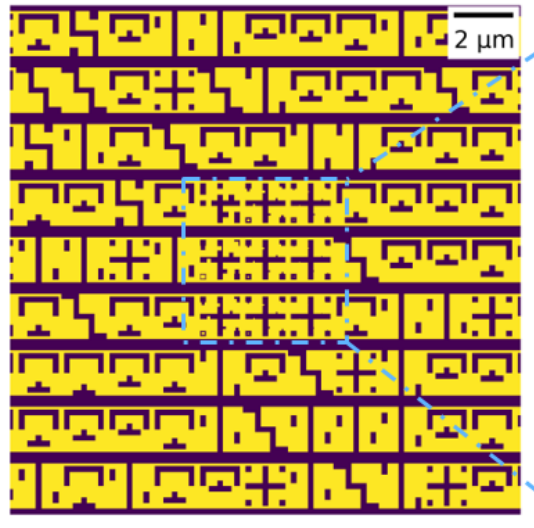

(2) ref mask center

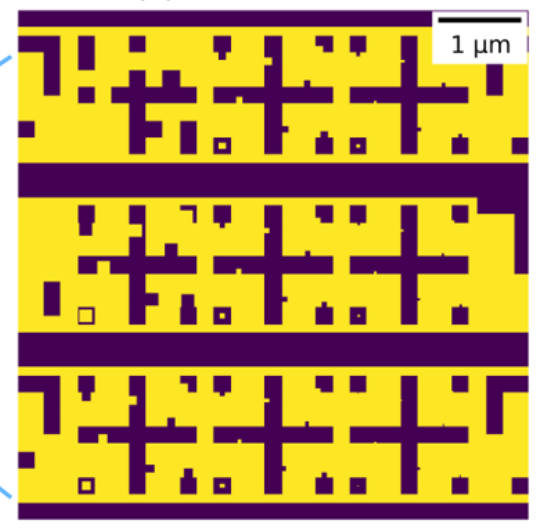

(3) Defect sizes

\begin{tabular}{|l|l|l|}
\hline 200 & 80 & 40 \\
\hline 150 & 60 & 30 \\
\hline 100 & 50 & 20 \\
\hline
\end{tabular}

$(\mathrm{nm})$

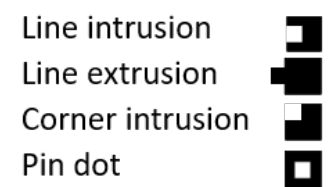

Figure 1. (1) The mask layout used in the present study (2) Zoom-in layout of the EUV mask at the central region and the types of programmed defects (3) Classification of the programmed defects by size in the nine regions in the center of the mask.

\section{Sample structure and pattern description}

The test mask sample is composed of HSQ (Hydrogen silsesquioxane) photoresist as an absorber with a thickness of $140 \mathrm{~nm}$ on top of a 40 pairs Mo/Si ML (multilayer). The programmed defects of various types are created with different sizes and located in the intricate logic pattern. The manufactured random logic pattern is shown in Fig. 1 (1). The critical dimension of the logic pattern is $200 \mathrm{~nm}$. In the middle of the field, we added programmed defects in nine regions with different sizes ranging from $200 \mathrm{~nm}$ (top-left) to $20 \mathrm{~nm}$ (bottom-right) in Fig. 1 (2). The sizes of the defects in the nine regions are classified in Fig. 1 (3). We note that the absorber pattern is not a state-of-the-art material for EUV masks and results in lower contrast than conventional materials. It implies that the maximum capability of RESCAN can be little higher than the results shown in this paper.

\section{Difference map (DM) reconstruction}

In the present study, we use the difference map (DM), which is a phase retrieval algorithm frequently used in ptychography [13]. Compared to the other algorithms, the DM method is a relatively robust reconstruction method against image noise and probe positions errors [12]. In this paper, we keep the parameters the same in the DM algorithm for an exact comparison and see the impact of the newly added correction algorithm explained in the following Section.

\section{Diffraction data correction (DDC) process}

Spatially, the diffraction pattern lies on the cosine plane [14]. If the incident angle is normal to the surface and sample-todetector distance $D$ is relatively large, one can use the conventional processing of the diffraction patterns for the reconstruction. However, the non-telecentricity of the imaging setup, i.e., the tilted incident angle, leads to a diffraction image distortion and thus has an impact on the reconstructed image quality. The diffraction distortion effect is more clearly visible when the object is a grating, which yields the conical diffraction pattern [14, 15]. This image distortion can be modified by employing a rotation matrix inversely [16]. In addition, exact diffraction patterns lie on a spherical detector, and a flat detector leads to a gradient intensity fall. Nevertheless, the image by the flat detector can be simply modified. The intensity falls as a function of $1 / r^{2}$, where $r$ is the distance from the sample to the detector. In the DM algorithm, farfield diffractions are used by a fast Fourier transform (FFT) for phase retrieval iterations. The FFT assumes that the diffraction image plane and the plane in the frequency domain are parallel. Thus, preprocessing algorithm trims the modified diffraction data to comply with the FFT rules. 


\section{RESULTS AND DISCUSSION}

\section{Reconstruction comparison and defect sensitivity}

We reconstructed areal images of the EUV mask with and without the diffraction data correction (DDC) process using the same dataset of the diffraction patterns and same reconstruction parameters. The obtained images of the EUV mask with and without the DDC are compared in Fig. 2. The image shown in Fig. 2 (1) is the reconstruction without the DDC process. In Fig. 2 (3), the corrected object is the one with the DDC process. Fig. 2 (2) shows the Fourier-frequency filtered image of the mask layout in the given optical setup for die-to-database comparison. Comparing the above images in detail, the line-extrusion defects on the horizontal lines are zoomed-in in Fig. 3 for different defect sizes in both conventional and corrected objects. The defects in the corrected objects displayed in Fig. 3 (2) are more visible than the defects in the conventional object in Fig. 3 (1).

(1) conv obj

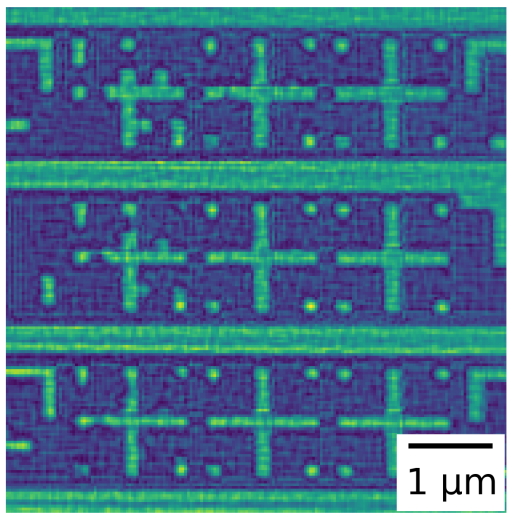

(2) refr

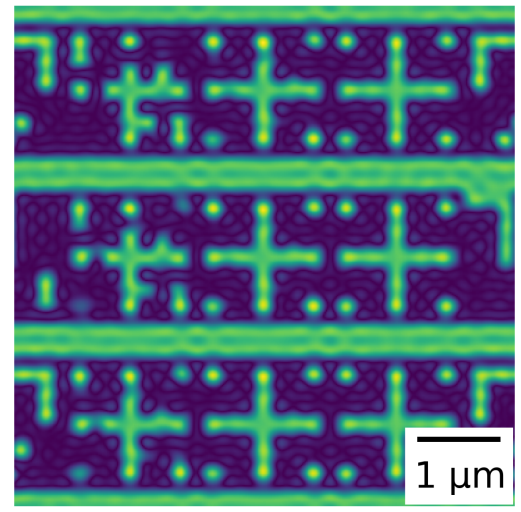

(3) corr obj

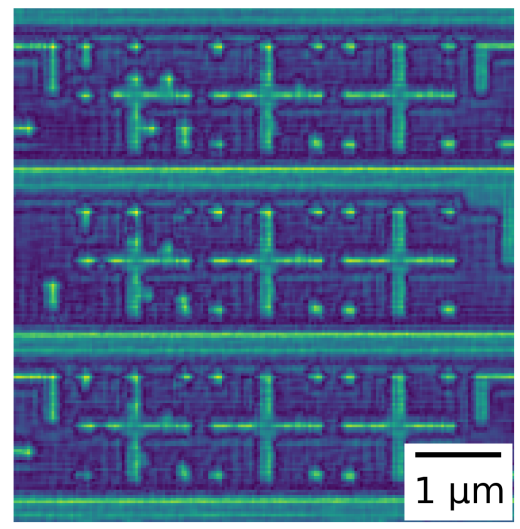

Figure 2. (1) the conventional object reconstructed without the DDC process. (2) Predicted aerial image of mask design (reference), and (3) the corrected object reconstructed with DDC process.

(1) Conv. Obj.



(2) Corr. Obj.



$80 \mathrm{~nm}$



$60 \mathrm{~nm}$

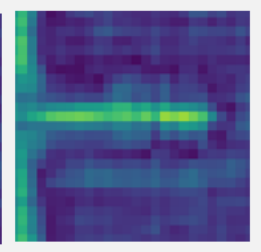

$50 \mathrm{~nm}$

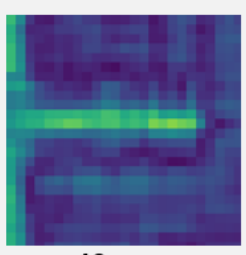

$40 \mathrm{~nm}$

Figure 3. Zoom-in images of Fig. 2 (1) and Fig. (3) for line-extrusion defects on the horizontal lines for different defect sizes: (1) reconstructed defect images in a conventional object, and (2) reconstructed defect images in corrected object for different defect sizes. 
(1) Defect signals on H-lines

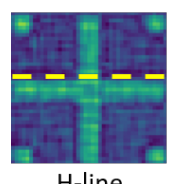

$\mathrm{H}$-line

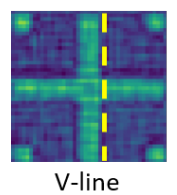

V-line
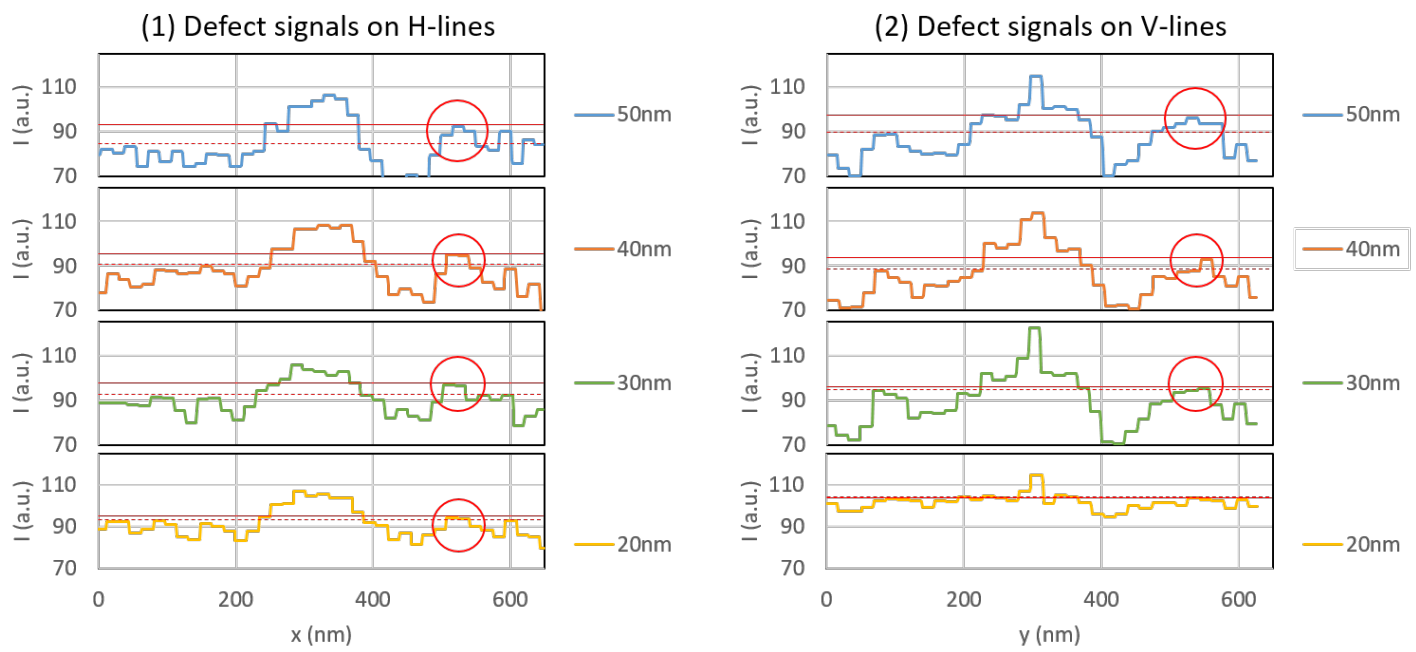

Figure 4. The cross-sectional profiles near the line-edges of the patterns: The defect signal curves are shown for the line-extrusion defects on both (1) horizontal (H-) and (2) vertical ( $\mathrm{V}$-) lines for different defect sizes from $50 \mathrm{~nm}$ to $20 \mathrm{~nm}$. The two attached images on the left side denote the locations of the cross-sections for $\mathrm{H}$ - and $\mathrm{V}$ - lines.

In Fig. 4, the intensity curves across the patterns to profile the line-extrusion defects in the corrected objects are shown. The line-extrusion defects on both horizontal and vertical lines (H-line and V-line, respectively) are measured near the line-edges. The intensity pops up where a defect is presented.

In Fig. 4, the two maximum intensity values in a curve are presented with red straight- and red dot lines, respectively. The red straight-lines denote the intensity maximum on the half-left side for $\mathrm{H}$-line and on the half-top side for V-line. These are the profiles near the line-edges that have no defects. On the other hand, the red dot-lines denote the intensity maximum that corresponds to the defect cross-sections near the line-edges, as shown on the half-right side of the H-line and halfbottom side of the V-line. The gap between the straight-line and the dot-line reveals the existence of the defect.

As a result, the defects on $\mathrm{H}$-lines were detected down to $20 \mathrm{~nm}$ while the defect on V-line were detected down to $30 \mathrm{~nm}$. The different defect sensitivities between $\mathrm{H}$ - and $\mathrm{V}$ lines might come from the 3D effect of the 140-nm-high absorber pattern and the mask settlement on a chuck against the illumination direction that can lead to the defect hide behind the linewidth. We would like to further demonstrate for the defect sensitivity in different circumstances (i.e., a line-extrusion defect located far away from the center in a large FOV) to generalize the capability of the current version of RESCAN. In fact, the defect sensitivity can be improved by testing the TaBN absorber of $70 \mathrm{~nm}$ height and 70 pairs Mo/Si ML mask sample, providing less 3D effect and higher contrast than the test mask in this study.

\section{Increased field of view}

In ptychography, the field of view (FOV) is defined by the scan size, which, in principle, can be as large as the computational infrastructure allows. However, image quality control is more difficult as the reconstructed image size is increased because the optical defocusing effect is increased as a function of the object field size. Figure 5 (1) shows the reconstructed image in the large FOV. The degree of defocus is increased in a direction away from the center that the programmed defects are located.

Here, we found that the DDC process removes the defocus gradient effect in all reconstructed fields. Fig. 5 (2) shows the reconstructed image with the DCC process. The defocus removal effect is clearly visible, comparing the two images in the top right corners. The reconstructed image data having a large FOV can provide benefits for users to manage the reconstructed image on their purpose in the APMI tool. For example, because the reconstructed image is modularized, it is possible to pick up a reconstructed image at a specific location in the mask based on their rule-of-thumb even though the full mask reconstruction computation is not finished yet. Besides, the large FOV image can be convenient in various 
situations. Further research for the DDC process will demonstrate better image quality, higher defect sensitivity, and larger FOV.

(1) conv obj

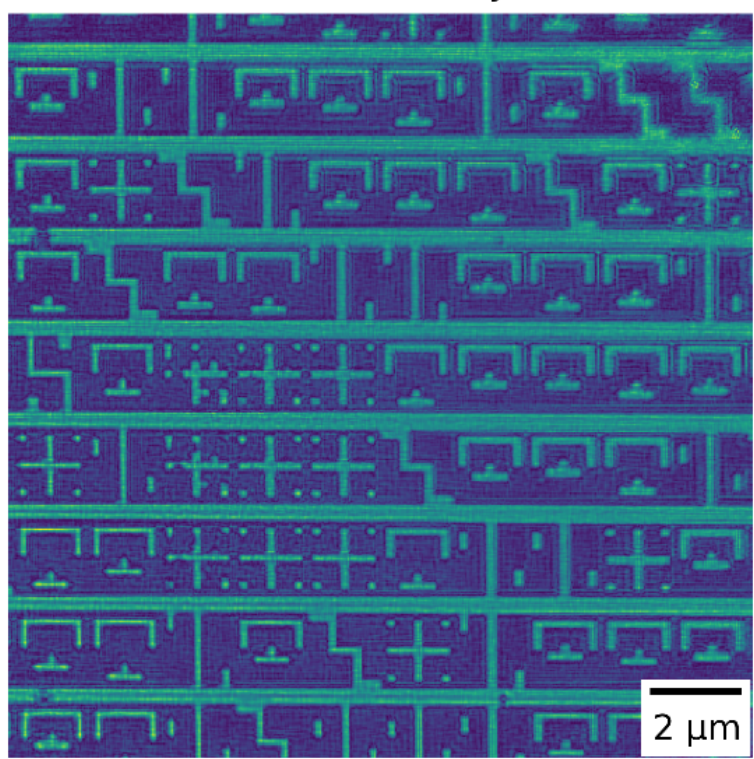

(2) corr obj

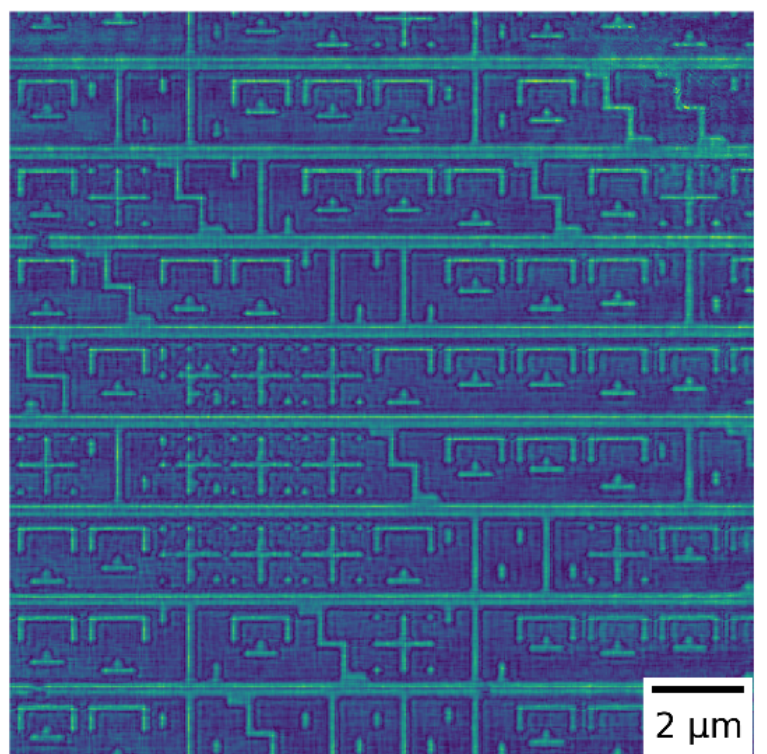

Figure 5. EUV mask images in a large FOV reconstructed (1) in a conventional way and (2) with the DDC processing, which results in an image with better defect sensitivity and without a focus gradient across the FOV.

\section{CONCLUSIONS}

The additional preprocessing that we implemented remaps the diffraction data on a sphere in the Fourier space and corrects the non-telecentricity of the imaging configuration. We demonstrated that by correcting the diffraction patterns recorded in RESCAN, we could significantly improve the defect sensitivity to enable the required EUV mask inspection technology for the 5-nm node. We demonstrated defect sensitivity down to $20 \mathrm{~nm}$. In fact, the defect resolution and sensitivity achieved in this study can be further improved by using commercial EUV masks, which have higher contrast (approx. 10\% higher) and less 3D effects (approx. 50\% less) than the test mask used in this study. In addition, the resolution can be further improved $10 \%$ more than the result shown here if the algorithm is optimized to use full CCD, which might lead to defect sensitivity $<18 \mathrm{~nm}$. Furthermore, this implemented correction method removes the focal gradient, and thereby the reconstructed image FOV is enlarged, which allows for flexible post data processing. These improvements can also be applied for our plan to upgrade the RESCAN for high NA APMI toward below $5 \mathrm{~nm}$ EUVL node [10].

\section{ACKNOWLEDGMENTS}

The authors would like to thank Markus Kropf, Michaela Vockenhuber, and Jose Gabadinho for their technical support at the XIL-II beamline. This project has received funding from the Electronic Component Systems for European Leadership Joint Undertaking under grant agreement No 783247-TAPES3. This Joint Undertaking receives support from the European Union's Horizon 2020 research and innovation program and the Netherlands, Belgium, France, Germany, Israel. Coauthors (A.T and R.N.) thank the Swiss National Science Foundation for financial support (SNF Grant Number: 200021_172768). Part of this research was performed at Swiss Light Source, Paul Scherrer Institut. 


\section{REFERENCES}

[1] S. Kim, R. Chalykh, H. Kim, S. Lee, C. Park, M. Hwang, J. Park, J. Park, H. Kim, J. Jeon, I. Kim, D. Lee, J. Na, J. Kim, S. Lee, H. Kim, S. Nam, "Progress in EUV lithography toward manufacturing," Proc. SPIE 10143, 1014306 (2017).

[2] E. Verduijn, P. Mangat, O. Wood, J. Rankin, Y. Chen, F. Goodwin, R. Capelli, S. Perlitz, D. Hellweg, R. Bonam, S. Matham, N. Felix, D. Corliss, "Printability and actinic AIMS review of programmed mask blank defects," Proc. SPIE 10143, 101430K (2017).

[3] T. Liang, Y. Tezuka, M. Jager, K. Chakravorty, S. Sayan, E. Frendberg, S. Satyanarayana, F. Ghadiali, G. Zhang, F. Abboud, "EUV mask infrastructure and actinic pattern mask inspection," Proc. SPIE 11323, 1132310 (2020).

[4] A. Tchikoulaeva, H. Miyai, T. Kohyama, K. Takehisa, H. Kusunose, "Enabling EUVL high-volume manufacturing with actinic patterned mask inspection," Proc. SPIE 11323, 113231K (2020).

[5] P. Helfenstein, I. Mohacsi, R. Rajendran, Y. Ekinci, "Scanning coherent diffractive imaging methods for actinic extreme ultraviolet mask metrology," J. Micro/Nanolith. MEMS MOEMS 15(3) 034006 (2016).

[6] I. Mochi, P. Helfenstein, R. Rajeev, S. Fernandez, D. Kazazis, S. Yoshitake, and Y. Ekinci, "Actinic inspection of EUV reticles with arbitrary pattern design," Proc. SPIE 10450, 1045007-1045016 (2017).

[7] I. Mochi, S. Fernandez, R. Nebling, U. Locans, P. Helfenstein. R. Rajeev, A. Dejkameh, D. Kazazis, L. Tseng, and Y. Ekinci, "Absorber and phase defect inspection on EUV reticles using RESCAN," Proc. SPIE. 10957, 109570W (2019).

[8] I. Mochi, D. Kazazis, L. Tseng, S. Fernandez, R. Rajeev, U. Locans, A. Dejkameh, R. Nebling, Y. Ekinci, "Lensless metrology for semiconductor lithography at EUV, "Proc. SPIE 11057, 1105703 (2019).

[9] I. Mochi, M. Timmermans, E. Gallagher, M. Mariano, I. Pollentier, R. Rajendran, P. Helfenstein, S. Fernandez, D. Kazazis, Y. Ekinci, "Experimental evaluation of the impact of carbon nanotube EUV pellicles on reticle imaging," J. Micro/Nanolith. MEMS MOEMS 18(1) 014002 (2019).

[10] I. Mochi, H. Kim, U. Locans, A. Dejkameh, R. Nebling, D. Kazazis, Y. Ekinci, "Illumination control in lensless imaging for EUV mask inspection and review," Proc. SPIE 11323, 113231I-1 (2020).

[11] J. Fienup, "Reconstruction of a complex-valued object from the modulus of its Fourier transform using a support constraint," J. Opt. Soc. Am. A 4(1) (1987).

[12]R. Nebling, I. Mochi, D. Kazazis, U. Locans, A. Dejkameh, Y. Ekinci, "EUV reticle inspection using phase retrieval algorithms: a performance comparison," Proc. SPIE. 11147, 111470R (2019).

[13] P. Thibault, M. Dierolf, O. Bunk, A. Menzel, F. Pfeiffer, "Probe retrieval in ptychographic coherent diffractive imaging," Ultramicroscopy 109(4), 338-343 (2009).

[14] J. Harvey, R. Pfisterer, "Understanding diffraction grating behavior: including conical diffraction and Rayleigh anomalies from transmission gratings," Opt. Eng. 58(8), 087105 (2019).

[15] D. Gardner, B. Zhang, M. Seaberg, L. Martin, D. Adams, F. Salmassi, E. Gullikson, H. Kapteyn, M. Murnane, "High numerical aperture reflection mode coherent diffraction microscopy using off-axis apertured illumination," Opt. Express 20 (17), 19050-19059 (2012).

[16] M. Seaberg, B. Zhang, D. Adams, D. Gardner, H. Kapteyn, M. Murnane, "Tabletop coherent diffractive imaging of extended objects in transmission and reflection geometry," Proc. SPIE 8851, 88510Y (2013). 